\title{
Short-Term Changes in Behaviors Resulting from COVID-19-Related Social Isolation and Their Influences on Mental Health in Ghana
}

\author{
Nestor Asiamah ${ }^{1}$ (D) . Frank Frimpong Opuni ${ }^{2} \cdot$ Edwin Mends-Brew $^{3} \cdot$ Samuel Worlanyo Mensah $^{4}$. \\ Henry Kofi Mensah ${ }^{5} \cdot$ Fidelis Quansah $^{6}$
}

Received: 3 May 2020 / Accepted: 1 October 2020 / Published online: 8 October 2020

(c) Springer Science+Business Media, LLC, part of Springer Nature 2020

\begin{abstract}
This study assessed the behavioral outcomes of Coronavirus 2019 (COVID-19) social distancing protocols and their influences on mental health. An online survey hosted by Survey Monkey was utilized to collect data from residents of three Ghanaian cities of Accra, Kumasi and Tamale. A total of 621 surveys were analyzed, with a sensitivity analysis utilized to select covariates for the regression model. The average age of participants was about 36 years. Findings indicate that reduced physical activity time and a change in sexual activity and smoking frequency are some short-term changes in behavior resulting from social isolation during the lockdown. An increase in sedentary behavior had a negative influence on mental health. For the most part, changes in behaviors in the short-term were associated with lower mental health scores. The study implied that COVID-19 social distancing measures should be implemented alongside public education for discouraging unhealthy changes in behaviors.
\end{abstract}

Keywords COVID-19 $\cdot$ Behavior $\cdot$ Health behavior $\cdot$ Social isolation $\cdot$ Mental health $\cdot$ Ghana

\section{Introduction}

Coronavirus 2019 (COVID-19) is a novel virus that was first detected in Wuhan City, China (Lewnard and Lo 2020; Lin et al. 2020). Over a period of $<5$ months (between December 2019 and April 2020), the virus grew from being a local epidemic in Wuhan to a fear-inspiring global pandemic. As of September 13, 2020, the virus had killed 920,795 out of

Nestor Asiamah

nestor.asiamah@ace-gh.org

Frank Frimpong Opuni

fofrimpong@atu.edu.gh

Edwin Mends-Brew

emendsbrew@yahoo.com

Samuel Worlanyo Mensah

samuelmensah4011@yahoo.com

Henry Kofi Mensah

hkmensah@knust.edu.gh

Fidelis Quansah

fidelis.quansah@upsamail.edu.gh

1 Africa Centre for Epidemiology, Department of Behavioral Epidemiology, Accra North, P. O. Box AN 16284, Accra, Ghana
28,787,808 people who tested positive for it (Johns Hopkins University 2020), making it one of the world's deadliest pandemics (Lin et al. 2020; Pung et al. 2020). COVID-19 can be considered a highly contagious virus not only because it has infected thousands of people but also because it has taken a relatively short time to spread to most regions of the world. Without appropriate measures to contain it therefore, COVID-19 could infect a third of the world in a year.

2 Department of Marketing, Accra Technical University, Accra, Ghana

3 Accra Technical University, Department of Statistics and Mathematics, Accra, Ghana

4 Department of Development and Economics, Wisconsin International University College, Accra, Ghana

5 Department of Human Resources \& Organizational Development, Kwame Nkrumah University of Science and Technology, Kumasi, Ghana

6 Faculty of Management Studies, University of Professional Studies Accra, Madina, Ghana 
As the case has been with previous pandemics, the nonavailability of a vaccine has allowed COVID-19 to spread and grow into a global pandemic. Since the testing and production of a potential vaccine for an infectious disease takes an average period of 24 months (Josefsberg and Buckland 2012; Anderson et al. 2020), measures to cut the chains of infection are the only way to contain COVID-19 in the short-term. One of such measures, which is considered the best way to contain an epidemic or pandemic in the absence of a vaccine (Lin et al. 2020; Pung et al. 2020), is the enforcement of social distancing protocols in affected regions. With COVID-19, the adoption of this approach is a global shared goal that has brought about a complete or partial lockdown of affected countries. As demonstrated by China with its lockdown of Wuhan, social distancing protocols are the ultimate weapon for fighting COVID-19 in the short-term. This notwithstanding, their socio-economic impacts can be dire (Brooks et al. 2020; Armitage and Nellums 2020). Economic impacts such as unemployment, the weakening of the foundation of the global economy characterized by the United States (US) and China, and a potential collapse of emerging economies, at least in the short-term, are frequently reported economic consequences of COVID-19 (Anderson et al. 2020; Lewnard and Lo 2020). Other consequences that are significant but seem underreported (Lewnard and Lo 2020; Armitage and Nellums 2020) are social implications such as public health decline that exacerbate the above economic losses. Social distancing mechanisms, for example, could curtail individual physical activity (PA) trajectories. It is also possible that social distancing will limit access to food and public services, especially in developing countries where citizens may be unable to afford basic needs such as food if socially isolated.

We argue based on the Fogg behavior model (FBM) proposed by Fogg (2009) that a pandemic such as COVID-19 and its sudden lockdowns are extreme events that would cause fear and panic as people try to cope with them. Per the disengagement theory of aging developed by Cumming and Henry (1961), anxiety and mental health struggles may result from a sudden lockdown because social disengagement is a gradual process that would overwhelm people who try to achieve it instantly or in the short-term. We are, therefore, of the view that social isolation necessitated by a COVID-19-related lockdown would not only cause fear and panic in the short-term but could also lead to anxiety and consequently a decline in mental health in the general population. Similarly, short-term social isolation can cause major changes in health behaviors that can increase the burden of disease and disability. This argument is corroborated by some researchers (Malcolm et al. 2019; Armitage and Nellums 2020) who have opined that a decline in mental health is the most likely consequence of social isolation caused by an unexpected event such as the outbreak of a disease. If so, stakeholders need to understand how significant changes in behaviors and their influences on mental health are and roll out suitable programs for avoiding or at least reducing public health risks that could be predicted by a COVID-19 lockdown. With some predicting the outbreak of a similar epidemic in future (Lewnard and Lo 2020; Li and Siegrist 2012) and others seeing the possibility of COVID-19 spreading for a long time (Li and Siegrist 2012; Pung et al. 2020), stakeholders need to understand changes in behaviors that could result from a lockdown as a precursor to designing appropriate programs for discouraging unhealthy changes in behaviors.

Possible COVID-19-related changes in behaviors have been acknowledged in the literature. The most frequently acknowledged changes are reduced PA and increased sedentary behavior due to limited access to the built environment and community services during the lockdown (Chen et al. 2020). On the other hand, the ability of exercise service providers to promptly move exercise classes online in response to social distancing measures have been reported (Chen et al. 2020; Jakobsson et al. 2020). As such, many individuals could exercise at home during the lockdown. Conspiracy theories have also indicated that alcohol intake, smoking, and the use of some substances (e.g. garlic) can protect the individual against COVID-19. Particularly in less educated populations, therefore, many individuals may take to substance use and smoking. Because families including couples may spend more time together at home during the lockdown (Fisher et al. 2020), sexual activity and domestic violence are also likely to increase due to social distancing measures. This study aimed to examine these changes and their influences on mental health. We understand that the aforesaid changes can be affected by demographic and individual characteristics. Education and income, for example, are likely to affect one's ability to utilize online exercise classes during the lockdown. For this reason, we adjusted for key covariates in testing the association between the said behavioral changes and mental health. Our choice of mental health as an outcome variable draws on commentaries indicating that mental health is the aspect of health most likely to be affected in the short-term by social distancing protocols (Serafini et al. 2020; Vindegaard and Eriksen Benros 2020). Our investigation was based on this primary research question: Do changes in behaviors due to COVID-19 social distancing measures have a significant influence on mental health?

\section{Methods}

\section{Design and Approach}

This study employed the descriptive correlational approach and online surveys targeting the general population. A cross-sectional analysis technique was adopted. The exclusive use of an online survey was the only way to collect data during the lockdown. 


\section{Population and Selection}

As this study was aimed at informing policy decisions for a specific region, the setting of this study was three cities (i.e. Greater Accra, Kumasi, and Tamale) affected by a COVID-19 mandatory lockdown in Ghana. The study population was individuals of the general population, preferably those aged 18 years or more, who were socially isolated as they complied with the mandatory lockdown. Participants were selected based on four inclusion criteria: (1) currently living in any of the cities facing mandatory lockdown; (2) having acquired at least a basic education instructed in English, the medium in which the survey was administered; (3) being in social isolation owing to the lockdown; and (4) willingness to participate in the study. The use of a powered sample (i.e. a sample determined based on a pre-determined statistical power and effect size) was not possible in this study for a couple of reasons. Firstly, we did not find any existing study that was based on our context. Secondly, we could not have used information from previous research to calculate a sample size because all existing studies applied substantially different methods. A deep look into the literature suggested that related webbased studies had utilized sample sizes ranging between $n=32$ and $n=4222$ to reach credible findings (Merolli et al. 2014; Balhara and Verma 2014; Liang et al. 2006). Considering our research approach and the geographical scope of the study setting, we hoped to achieve a sample size between 250 and 700 .

\section{Structure of the Online Survey}

The survey was developed by the researchers and hosted on Survey Monkey, a free survey creation platform that allows data sharing and analysis between research team members. It was chosen because of the researchers' ample experience with it and the fact that it provides user-friendly data transfer and analysis tools. The survey was developed from scratch, as opposed to using a template, because no existing template was suited for our study. The survey comprised 23 multiple-choice questions and a question introducing the mental health measure. The first question included the ethics statement and instructions for completing the survey. The next two questions (i.e. Q2 and Q3) screened for individuals who did not meet the inclusion criteria. Questions 4-8 and 13 captured demographic variables and covariates. Changes in behaviors were measured with questions 9-12 as well as 14-23. Question 24 presented the 9-item mental scale measure. The 'one question per page' design option that comes with the most legible text (Regmi et al. 2017) was chosen.

\section{Survey Development, Validation, and Piloting}

The survey was developed after the researchers discussed with two groups on what could be the ideal measures of mental health and changes in behaviors in the context of the study. The first group, which included four of the authors, was a WhatsApp-based group made up of research fellows of a Center of Excellence. Members, through the use of text messages and audio recordings, suggested potential measures for the study. Over Skype, the researchers then consulted with the second group, comprising two psychometricians and a statistician, to agree on an initial list of items for the survey. The lead researcher then developed a questionnaire of the items proposed. Following this, 10 copies of the questionnaire in sealed envelopes were sent through a private courier to individuals aged 24 years or more who had agreed to complete it in the neighborhood of the lead researcher. This step was part of the survey piloting arrangement. Over 2 days, questionnaires were completed and returned by 8 out of the 10 participants through the courier. Respondents commented on ambiguities and wording problems associated with the questionnaire. Through a voice call, the lead researcher contacted the participants to confirm and better understand the issues reported, enabling the researchers to further improve the wording of the items. A major change made to the instrument was replacing the word 'self-isolation' with 'social isolation' in most of the measures. An online survey of the final items (including an ethics statement) was then developed and piloted online with 10 different participants (WhatsApp $=4$; Facebook $=5$; Twitter $=1$ ). With no issues identified in the second pilot study, we sent the survey back to the two psychometricians consulted earlier for approval.

\section{Variable Selection and Operationalization}

This study focused on possible short-term changes in behaviors resulting from COVID-19-related social isolation or fears. Changes in smoking frequency, alcohol intake, and substance dependence were incorporated into the study owing to fake news about the possibility of smoking and the dependence on some substances (e.g. garlic, alcohol, marijuana) protecting against COVID-19. The other changes in behaviors were considered because they could be encouraged by social isolation. Table 1 shows a summary of all changes in behaviors and their operationalization. As Table 1 indicates, categorical variables were dummy-coded and one of their levels (categories) set as the reference. The table also shows underlying health conditions and demographic variables that, per existing studies (Sederer 2016; Lund et al. 2018), can confound the primary relationships of interest. Mental health was measured with a 9-item standard scale (with descriptive anchors strongly disagree - 1; disagree-2; 


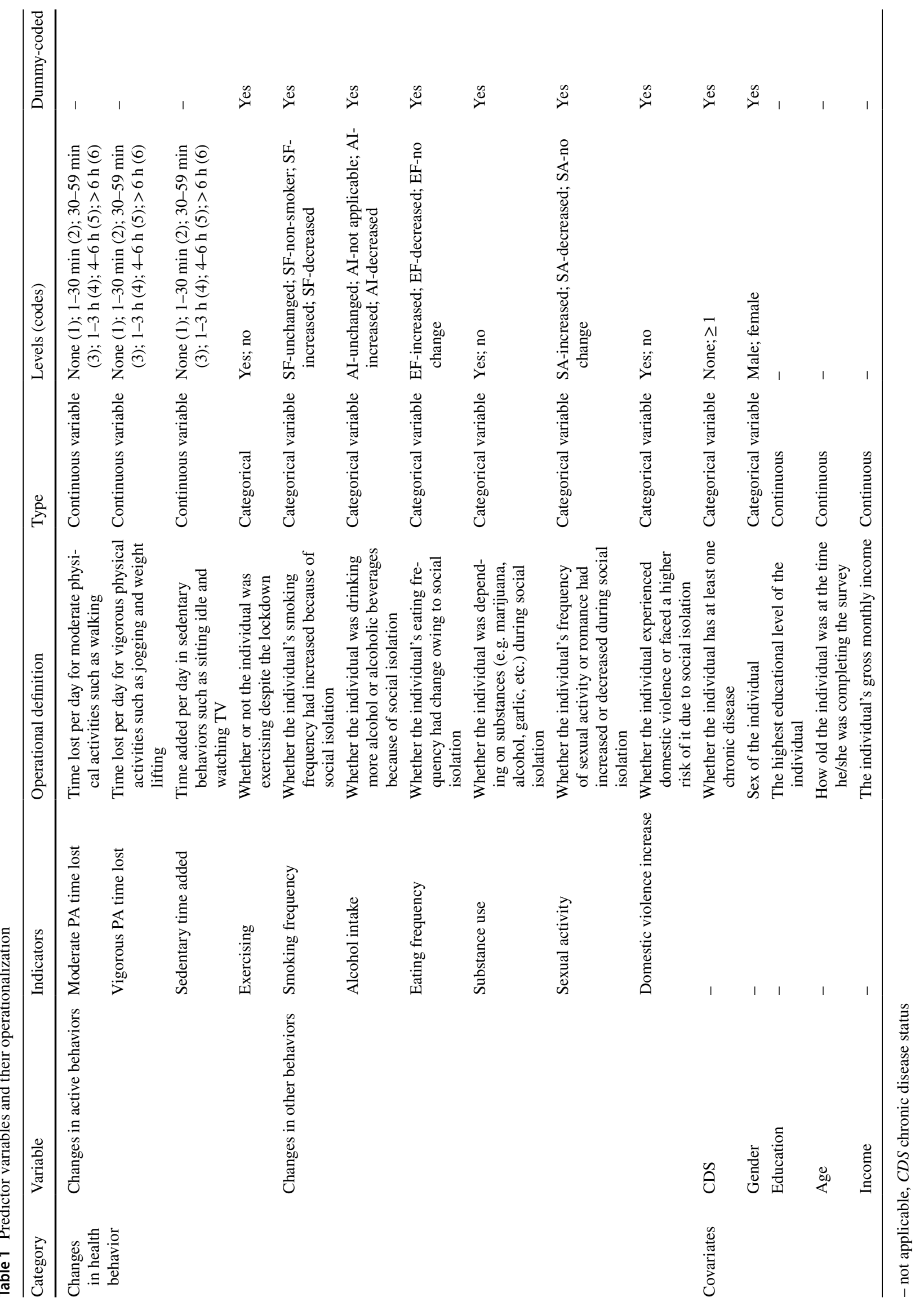


somewhat agree - 3; agree - 4; and strongly agree - 5) from Lukat et al. (2016). This tool is a unidimensional scale that produced satisfactory psychometric properties (including a Cronbach's $\alpha$ coefficient $=0.93$ ) on a sample representing the general population. It was preferred to other mental health measures because it has been properly validated for the general population and is the most holistic mental health measure (Lukat et al. 2016). In the current study, it produced a satisfactory Cronbach's $\alpha$ coefficient of 0.82 . Scores on the mental health measure were generated in harmony with the Lukat and colleagues; items were 'parceled' by adding them up. Appendix Table 5 shows items of the mental health measure used.

\section{Data Collection and Ethical Steps}

This study received ethical clearance from an institutional ethics review committee (\# 0012020-ACE) after the research protocol and ethical statement were reviewed by the committee. In agreement with best practices, we ensured that the first question of the survey presented the ethical statement (Merolli et al. 2014; Balhara and Verma 2014), which means that only individuals who agreed to participate voluntarily (by ticking 'Yes') completed the survey. The ethical statement indicated the purpose and importance of the study as well as the risk-free nature of our data collection process. The inclusion criteria and instructions for completing the survey were also presented as aspects of the ethical statement. We created different versions of the survey that could easily be completed on all social media platforms including WhatsApp. We published the survey a week after the lockdown by sending a link of it to all our contacts using WhatsApp and asking them to complete the questionnaire and share it with their contacts. Thus, snowball selection was applied to distribute the survey. Subsequently, the researchers, through their personal accounts, published the link on Facebook, Twitter, LinkedIn and other social media platforms. The shared link took the participant to a pop-up questionnaire that could be completed even with a relatively weak internet network. Participants did not have to download the survey before completing it. The survey was distributed and completed over about 2 weeks (April 4-16, 2020) and was closed on April 16, 2020. Its average completion time was about $7 \mathrm{~min}$. We programed the survey at Survey Monkey to prevent multiple responses from the same participant. For further research purposes, we designed the survey to allow individuals outside the study setting to respond. We did not provide incentives for participation.

\section{Statistical Analyses Method}

Data in a Microsoft (MS) Excel format were downloaded from Survey Monkey. Coding was done in MS Excel and the resulting data transported to SPSS version 25 (IBM Inc., NY, USA), which was used for data analysis. Descriptive statistics (frequency and per cent) were used to summarize the data after five questionnaires with missing items were discarded in line with the recommendation of Garson (2012). The Shapiro-Wilk's test was performed to screen for outliers and confirm normality of data of mental health (Garson 2012). This test on the data confirmed normality $(p=0.294)$ and the absence of outliers. Pearson's correlation test was then used to assess bivariate correlations between the variables. A multiple linear regression model was fitted to assess the influence of the changes in behaviors on mental health, with potential confounding variables adjusted for. Before fitting the regression model, a sensitivity analysis was conducted to screen for relevant potential confounding variables in harmony with the procedure adopted elsewhere (Rothman and Greenland 1998; Rezai et al. 2008). In this analysis, univariate regression models were used to estimate crude coefficients (i.e. standardized and unstandardized coefficients and their $95 \%$ confidence intervals) indicating the influence of the covariates and changes in behaviors on mental health. Covariates with $\mathrm{p}>0.25$ were removed and those with $\mathrm{p} \leq 0.25$ were kept for the second level of the sensitivity analysis. At this stage, only chronic disease status (CDS) was removed. At the second level, multiple linear regression models were fitted to estimate coefficients (including their 95\% confidence intervals) representing the influences of changes in behavior and each of the remaining covariates on mental health. Any covariate that led to a $10 \%$ change (decrease or increase) in the coefficients of the behaviors from the first level was kept and incorporated into the final regression model (see Table 3 ) as a covariate. At this stage, age and income were removed.

\section{Findings}

\section{Response Rate, Demographic Characteristics, and Changes in Behaviors}

We achieved a survey completion rate of $100 \%$, which means all participants $(n=643)$ completed the survey. After applying the inclusion criteria, 22 questionnaires were dropped. Of the 621 remaining questionnaires analyzed, $55 \%(n=342)$ were completed by residents of Accra, $25 \%(n=157)$ by residents of Kumasi; and 20\% $(n=122)$ by residents of Tamale. As Table 2 indicates, about $35 \%(n=215)$ of participants were female and $65 \%(n=406)$ were male. About $94 \%$ of participants $(n=586)$ had tertiary education, which means that most of the sample had a high education. The age of participants ranged between 18 and 64 years. Table 2 shows some dramatic changes in behaviors. That is, $80 \%$ of participants lost moderate physical activity time, with over 
Table 2 Summary statistics on covariates and changes in behaviors

\begin{tabular}{|c|c|c|c|c|}
\hline Category & Variable & Levels (groups) & $n$ & Per cent $(\%)$ \\
\hline \multirow{15}{*}{$\begin{array}{l}\text { Demographic vari- } \\
\text { ables and covariates }\end{array}$} & \multirow[t]{2}{*}{ Gender $(N=621)$} & Female & 215 & 34.6 \\
\hline & & Male & 406 & 65.4 \\
\hline & \multirow[t]{2}{*}{ Educational level $(n=621)$} & Secondary level & 35 & 5.6 \\
\hline & & Tertiary level & 586 & 94.4 \\
\hline & \multirow[t]{5}{*}{ Age $(N=621)$} & $18-24$ years & 110 & 17.7 \\
\hline & & 25-34 years & 213 & 34.3 \\
\hline & & $35-44$ years & 143 & 23 \\
\hline & & $45-54$ years & 120 & 19.3 \\
\hline & & $55-64$ years & 35 & 5.6 \\
\hline & \multirow[t]{4}{*}{ Income $(N=621)$} & None & 105 & 16.9 \\
\hline & & $<500$ & 50 & 8.1 \\
\hline & & $500-1000$ & 115 & 18.5 \\
\hline & & $>1000$ & 351 & 56.5 \\
\hline & \multirow[t]{2}{*}{$\operatorname{CDS}(N=621)$} & None & 556 & 89.5 \\
\hline & & $\geq 1$ & 65 & 10.5 \\
\hline \multirow[t]{35}{*}{ Changes in behavior } & \multirow[t]{6}{*}{ MPA time lost $(N=621)$} & None & 124 & 20.0 \\
\hline & & $1-30 \mathrm{~min}$ & 45 & 7.20 \\
\hline & & $30-59 \mathrm{~min}$ & 73 & 11.8 \\
\hline & & $1-3 \mathrm{~h}$ & 174 & 28.0 \\
\hline & & $4-6 \mathrm{~h}$ & 75 & 12.1 \\
\hline & & $>6 \mathrm{~h}$ & 130 & 20.9 \\
\hline & \multirow[t]{6}{*}{ VPA time lost $(N=621)$} & None & 184 & 29.6 \\
\hline & & $1-30 \mathrm{~min}$ & 65 & 10.5 \\
\hline & & $30-59 \min$ & 144 & 23.2 \\
\hline & & $1-3 \mathrm{~h}$ & 158 & 25.4 \\
\hline & & $4-6 \mathrm{~h}$ & 35 & 5.6 \\
\hline & & $>6 \mathrm{~h}$ & 35 & 5.6 \\
\hline & \multirow[t]{6}{*}{ Sedentary behavior time added $(N=621)$} & None & 114 & 18.4 \\
\hline & & $1-30 \mathrm{~min}$ & 35 & 5.6 \\
\hline & & $30-59 \min$ & 49 & 7.9 \\
\hline & & $1-3 \mathrm{~h}$ & 153 & 24.6 \\
\hline & & $4-6 \mathrm{~h}$ & 150 & 24.2 \\
\hline & & $>6 \mathrm{~h}$ & 120 & 19.3 \\
\hline & \multirow[t]{2}{*}{ Exercising $(N=621)$} & Yes & 397 & 63.9 \\
\hline & & No & 224 & 36.1 \\
\hline & \multirow[t]{2}{*}{ Smoking frequency $(N=621)$} & SF-unchanged & 173 & 27.9 \\
\hline & & SF-non-smoker & 448 & 72.1 \\
\hline & \multirow[t]{3}{*}{ Alcohol intake $(N=621)$} & AI-increased & 19 & 3.1 \\
\hline & & AI-unchanged & 164 & 26.4 \\
\hline & & AI-not applicable & 438 & 70.5 \\
\hline & \multirow[t]{3}{*}{ Eating frequency $(N=621)$} & EF-decreased & 75 & 12.1 \\
\hline & & EF-increased & 262 & 42.2 \\
\hline & & EF-no change & 284 & 45.7 \\
\hline & \multirow[t]{2}{*}{ Substance use $(\mathrm{N}=621)$} & No & 571 & 91.9 \\
\hline & & Yes & 50 & 8.1 \\
\hline & \multirow[t]{3}{*}{ Sexual activity $(\mathrm{N}=621)$} & SA-decreased & 125 & 20.1 \\
\hline & & SA-increased & 77 & 12.4 \\
\hline & & SA-no change & 419 & 67.5 \\
\hline & \multirow[t]{2}{*}{ Domestic violence increase $(n=621)$} & No & 571 & 91.9 \\
\hline & & Yes & 50 & 8.1 \\
\hline
\end{tabular}

$C D S$ chronic disease status, $M P A$ moderate physical activity, $V P A$ vigorous physical activity, $N$ total sample analyzed, $n$ frequency 
$50 \%$ losing at least $1 \mathrm{~h}$ of moderate physical activity time. Similarly, more than $50 \%$ added at least $1 \mathrm{~h}$ to their sedentary behavior time. About $64 \%(n=397)$ of participants were exercising during the lockdown, whereas there was no change in the frequency of smoking for $28 \%(n=173)$ of participants who were smokers. The frequency of alcohol intake increased for 3\% $(n=19)$ and did not change for $26 \%$ $(n=164)$ of participants. The frequency of eating decreased for $12 \%(n=75)$ and increased for $42 \%(n=262)$ of participants. About $8 \%(n=50)$ of participants agreed they used substances to protect themselves against COVID-19 and faced a higher risk of domestic violence. Sexual activity decreased for $20 \%(n=125)$ and increased for $12 \%(n=77)$ of participants. Appendix Table 6 shows a distribution of average mental health scores across categorical predictors.

\section{The Influences of Changes in Behaviors on Mental Health}

Table 3 shows some significant correlations at $p<0.001$ and $\mathrm{p}<0.05$. For example, MPATL and mental health are negatively correlated $(r=-0.235 ; \mathrm{p}<0.001$; two-tailed). This result connotes that mental health decreases as moderate physical activity time lost increases. Table 4 shows regression coefficients resulting from this and other correlation coefficients in Table 3. It can be seen that moderate PA time lost makes a significant influence on mental health $(\beta=-0.07 ; t=-3.28, \mathrm{p}<0.05)$, suggesting that mental health decreased with an increase in moderate PA time lost. Sedentary behavior time added also made a negative influence on mental health $(\beta=-0.85 ; t=-46.76, \mathrm{p}=0.000)$. The influence of 'SF-unchanged' is about 2 times lower compared with that of 'SF-non-smokers' $(B=-2.05$; $t=-4.97, \mathrm{p}=0.000$ ), which indicates that the mental health of individuals who did not have a change in their smoking frequency was lower compared with non-smokers. The influence of 'AI-not applicable' on mental health is about 2 times lower compared with that of 'AI-increased' $(\mathrm{B}=-2.34 ; t=-5.76, \mathrm{p}=0.000)$, implying that individuals who did not drink alcohol at all reported lower mental health scores compared with those whose frequency of alcohol intake decreased. The influence of 'AI-unchanged' on mental health is about 0.7 times higher compared with that of 'AI-decreased' ( $\mathrm{B}=0.695 ; t=4.26, \mathrm{p}=0.000)$, indicating that individuals whose frequency of alcohol intake remained the same reported larger mental health scores compared with those who had a decrease in their frequency of alcohol intake. An increase in eating frequency (i.e. EF-increase) and a decrease in eating frequency (i.e. EF-decrease) were both associated with lower influences on mental health compared with 'EF-no change'. That is, compared with those whose eating frequency remained the same, individuals whose eating frequency increased or decreased reported lower mental health scores. Those who used substances to protect themselves against COVID-19 had better mental health compared with those who did not $(\mathrm{B}=1.55 ; t=3.08$, $\mathrm{p}<0.05$ ). Finally, those with increased sexual activity (i.e. SA-increase) had better mental health compared with those whose sexual activity level did not change $(\beta=2.44 ; t=5.75$, $\mathrm{p}=0.000$ ). The independence-of-errors and multicollinearity assumptions were met based on tolerance $\geq 0.1$ (for each predictor) and Durbin-Watson $=1.65$ (for the regression model) in Table 4 (Garson 2012).

\section{Discussion}

A major change in behavior resulting from COVID-19-related social isolation is a reduction in physical activity and an increase in sedentary behavior time. This outcome is consistent with studies (Mata-Lima et al. 2013; Martin 2015; Armitage and Nellums 2020) that have confirmed or reported a decline in PA and an increase in sedentary behavior as a result of social isolation caused by natural events including wars, earthquakes, and epidemics. A loss of moderate PA time was further found to make a negative influence on mental health, which suggests that mental health reduces in the sample as moderate PA time lost increases and sedentary behavior time added increases. Supporting this evidence is the systematic review of Hoare et al. (2016) indicating that mental health reduces with PA and an increase in sedentary behavior time. The empirical study of Ellingson et al. (2018) conducted in the US also backs our result with its evidence that a loss in moderate PA time and an increase in sedentariness is negatively associated with mental health in young adults. More so, several studies (Walsh 2011; Allen et al. 2014; Shim et al. 2014; Lund et al. 2018) reported a negative influence of reduced PA or increased sedentary behavior on mental health in the general population. Unlike previous pieces of evidence however, our result is the first linked to social isolation driven by a pandemic. A noteworthy connotation of our result is that social distancing measures should be rolled out with PA promotion programs to encourage indoor PA during social isolation. It may thus be necessary for governments to intensify PA counseling via the media before and during a lockdown. As done in parts of the UK (Mytton et al. 2012; Anderson et al. 2020), gyms and parks should be considered essential service providers and allowed to operate during a lockdown. However, a strict observance of social distancing protocols at these PA centers is imperative.

Despite the strong force with which conspiracy theorists used the social media to promote smoking as a behavior that protects against COVID-19 (Li and Siegrist 2012; Anderson et al. 2020), this study did not find any change in smoking among socially isolated participants. Based on 


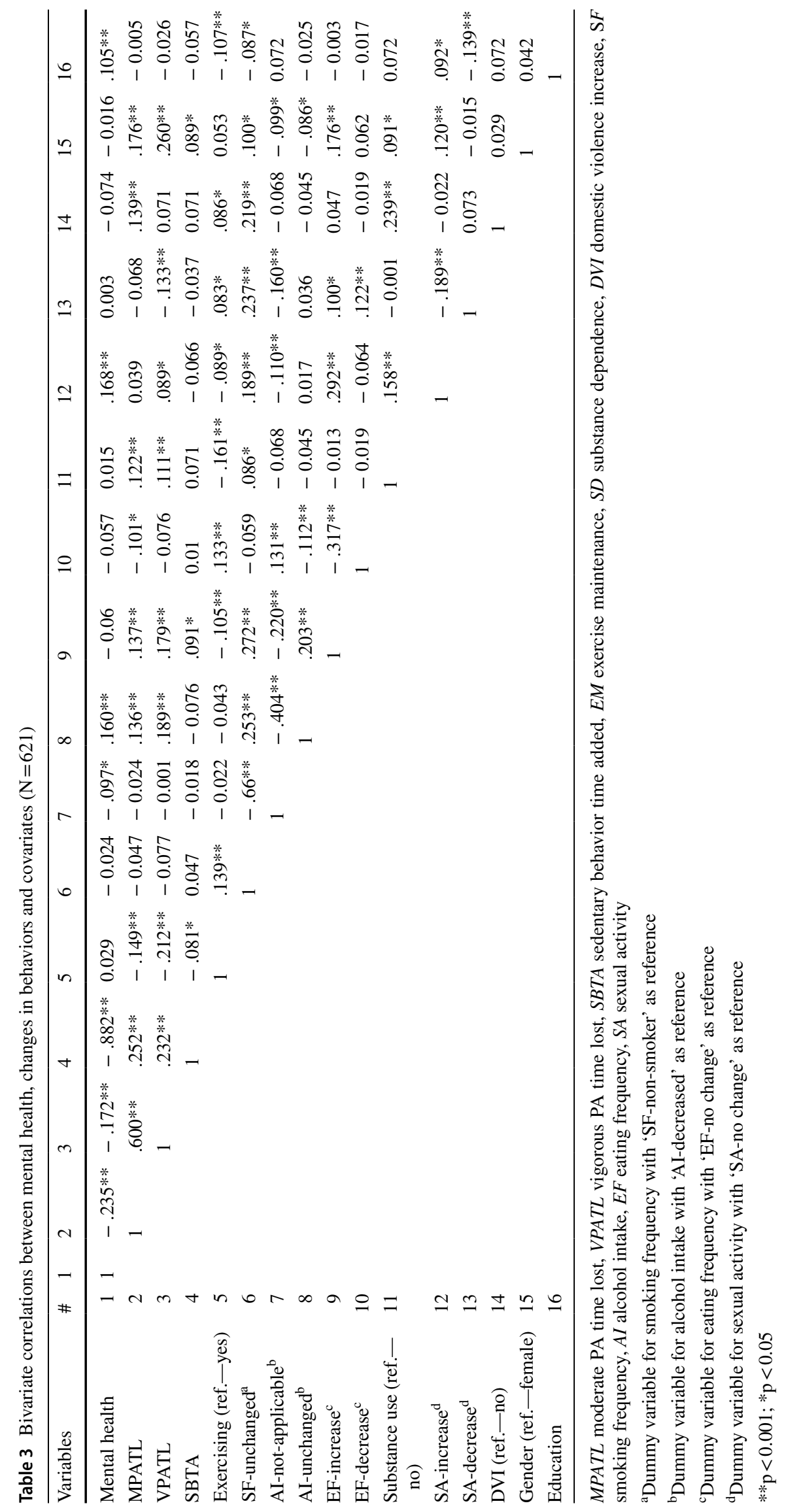


Table 4 The association between mental health, changes in health behaviors, and covariates $(\mathrm{N}=621)$

\begin{tabular}{|c|c|c|c|c|c|c|}
\hline \multirow[t]{2}{*}{ Variables } & \multicolumn{4}{|c|}{ Coefficients } & \multirow[t]{2}{*}{$95 \% \mathrm{CI}$} & \multirow[t]{2}{*}{ Tolerance } \\
\hline & B & S.E. & $\beta$ & $t$ & & \\
\hline \multicolumn{7}{|l|}{ Main influences } \\
\hline (Constant) & 42.526 & 1.755 & - & $24.228^{* *}$ & \pm 6.894 & - \\
\hline MPATL & -0.297 & 0.091 & -0.072 & $-3.279^{*}$ & \pm 0.356 & 0.603 \\
\hline VPATL & 0.072 & 0.111 & 0.015 & 0.65 & \pm 0.437 & 0.545 \\
\hline SBTA & -3.593 & 0.077 & -0.853 & $-46.755^{* *}$ & \pm 0.302 & 0.868 \\
\hline Exercising (ref.-yes) & -0.151 & 0.279 & -0.010 & -0.543 & \pm 1.095 & 0.847 \\
\hline SF-unchanged $^{\mathrm{a}}$ & -2.045 & 0.412 & -0.125 & $-4.965^{* *}$ & \pm 1.617 & 0.453 \\
\hline AI-not applicable ${ }^{\mathrm{b}}$ & -2.341 & 0.407 & -0.147 & $-5.756^{* *}$ & \pm 1.598 & 0.441 \\
\hline AI-unchanged ${ }^{\mathrm{b}}$ & 0.695 & 0.163 & 0.086 & $4.257 * *$ & \pm 0.641 & 0.709 \\
\hline EF-increase ${ }^{c}$ & -0.747 & 0.300 & -0.051 & $-2.484 *$ & \pm 1.181 & 0.688 \\
\hline EF-decrease ${ }^{c}$ & -0.987 & 0.418 & -0.044 & $-2.361 *$ & \pm 1.642 & 0.817 \\
\hline Substance use (ref.-no) & 1.545 & 0.502 & 0.058 & $3.080 *$ & \pm 1.970 & 0.814 \\
\hline SA-increase ${ }^{\mathrm{d}}$ & 2.441 & 0.425 & 0.111 & $5.749 * *$ & \pm 1.668 & 0.774 \\
\hline SA-decrease ${ }^{\mathrm{d}}$ & 0.186 & 0.340 & 0.010 & 0.548 & \pm 1.334 & 0.818 \\
\hline DVI (ref.-no) & 0.058 & 0.514 & 0.002 & 0.114 & \pm 2.019 & 0.774 \\
\hline \multicolumn{7}{|l|}{ Covariate influences } \\
\hline Gender (ref.-female) & 1.015 & 0.286 & 0.067 & $3.545^{* *}$ & \pm 1.125 & 0.816 \\
\hline Education & 1.282 & 0.552 & 0.041 & $2.323 *$ & \pm 2.167 & 0.936 \\
\hline \multicolumn{7}{|l|}{ Model fit } \\
\hline $\mathrm{R}^{2}$ & 0.825 & & & & & \\
\hline Adjusted $\mathrm{R}^{2}$ & 0.821 & & & & & \\
\hline $\mathrm{R}^{2}$ change & 0.004 & & & & & \\
\hline Durbin-Watson & 1.646 & & & & & \\
\hline $\mathrm{F}$ & $178.54 * *$ & & & & & \\
\hline
\end{tabular}

$B$ unstandardized coefficient, $\beta$ standardized coefficient, $C I$ confidence interval, S.E. standard error (of B), SBTA sedentary behavior time added, MPATL moderate physical activity time lost, VPATL vigorous physical activity time lost, $S F$ smoking frequency, $A I$ alcohol intake, $E F$ eating frequency, $S A$ sexual activity, DVI domestic violence increase

a'Dummy variable for smoking frequency with 'SF-non-smoker' as reference

bDummy variable for alcohol intake with 'AI-decreased' as reference

'Dummy variable for eating frequency with 'EF-no change' as reference

${ }^{\mathrm{d}}$ Dummy variable for sexual activity with 'SA-no change' as reference

$* * \mathrm{p}<0.001 ; * \mathrm{p}<0.05$ commentaries in the literature (Jones-Jang et al. 2019), we argue that this result may be due to the fact that over $94 \%$ of the sample were highly educated individuals who may not yield to unfounded claims. Besides, smokers whose frequency of smoking did not change reported lower mental health scores than their colleagues who never smoked. This finding endorses previous studies that have found a negative relationship between smoking and mental health (Lawrence et al. 2009; Walsh 2011; Allen et al. 2014; Shim et al. 2014; Lund et al. 2018). Coupled with other distressing conditions caused by social distancing during the spread of COVID-19, our result may be an indicator of an intensified consequence of smoking. In any case, campaigns discouraging smoking and related behaviors during a lockdown ought to be intensified. Since the primary weapon for fighting many infectious diseases such as COVID-19 is the individual's immunity, behaviors such as smoking that have the tendency of weakening or disabling the immune system (Lawrence et al. 2009; Lund et al. 2018) must be eschewed at the individual level. For this reason, there is no alternative to conscientizing smokers regarding the health risks of smoking, particularly for those socially isolated.

A key change in behavior associated with COVID-19-related social isolation is a decrease and increase in the frequency of alcohol intake. Interestingly, those who did not drink alcohol at all reported smaller mental health scores compared to those who maintained their frequency of alcohol intake. This outcome tends to support previous empirical studies (German and Walzem 2000; Kaplan et al. 2000; Chiva-Blanch and Badimon 2020) confirming a positive 
influence of moderate alcohol intake on health. Also shedding light on this finding is the gradual growth in empirical research evidence indicating that wine, beer and other alcoholic beverages have properties that protect the individual against cardiovascular diseases and cognitive disorders (German and Walzem 2000; Lindberg and Amsterdam 2008). Even so, researchers (Kaplan et al. 2000; Chiva-Blanch and Badimon 2020) have warned that excessive consumption of alcohol increases the risk of disease. This being so, an increase in the frequency of alcohol intake can cause a major public health concern, especially for those forced into social isolation owing to the spread of an infectious disease such as COVID-19.

A change in the frequency of eating as a result of social isolation is a key finding of this study. While a fall in eating frequency may be due to poor access to supermarkets and supplies, an increase is possible for the working class or managerial elites with abundant food supplies. As social isolation during the lockdown compelled individuals to spend more time at home, an increase in the frequency of eating among those with enough savings is likely. This can be said of most of our participants who were highly educated and had a regular income. Further to the above, an increase and decrease in the frequency of eating were associated with lower mental health scores, logically because an increase translated into abuse of food while a decrease resulted in malnutrition in the short-term. This thinking squares with studies (Prentice 2001; Fuhrman 2018) that have revealed that food can only confer its nutritional and health benefits when consumed in moderation. Moreover, short-term side effects of over- and/or under-eating include mental health struggles that can compel individuals to poorly rate their mental health (Prentice 2001; Fuhrman 2018). With these possibilities in view, programs for conscientizing residents facing a lockdown would have to be cognizant of potential changes in dietary behaviors.

A segment of our sample used substances (e.g. garlic, ginger) to protect themselves against COVID-19 during social isolation, which points to the likelihood that people were influenced by fake news regarding the protective properties of eating garlic, ginger, and other substances against COVID-19. More interestingly, those who used these substances reported higher mental health scores, an outcome that tends to add weight to claims that garlic, ginger, and similar substances have anti-inflammatory properties and therefore enhance the immune system and confer other health benefits (Arreola et al. 2015; Percival 2016). We would want to reason that the foregoing result was possibly driven by participants' psychological reaction to using substances to protect themselves against COVID-19. That is, dependence on substances may have boosted the confidence of participants in their health and consequently made them to overrate their mental health. Whether substance use was well-fated or a guise, it is understandable that people are likely to use unprescribed substances during the lockdown to protect themselves against COVID-19. Regardless of its impact on mental health in this study, substance use could mar individual and public health, thereby causing disabilities that may cost governments a fortune to rehabilitate.

Over the years, empirical research has produced mixed findings regarding the influence of sex on mental health (Bennett 2000; Galinsky and Waite 2014), but researchers, from a psychology perspective, have reasoned based on different scenarios that mental health could improve with sexual activity (Ganong and Larson 2011). Congruent with this stance is our result indicating an increase in mental health in those whose sexual activity increased. A common explanation to a positive influence of sexual activity on mental health is that sexual intercourse and romance increase individuals' happiness and satisfaction with life if they satisfy the individuals emotional needs (Bennett 2000; Galinsky and Waite 2014). We opine based on this argument that sexual activity would make a positive short-term influence on mental health during COVID-19-related social isolation. We would want to premise this stance around the idea that sexual activity in the early days of the lockdown may have provided total emotional satisfaction possibly because most participants, who make up an elite working class, did not have enough time for sex before the lockdown. As such, the participants had unsatisfied sexual needs before they went into social isolation. Psychologists explain that an increase in sexual activity to meet one's unsatisfied sexual needs always produces mental health benefits (Galinsky and Waite 2014).

Drawing on the foregoing assertion, we admit that changes in behaviors and their influences on mental health may differ in the long run when people may successfully adapt to the lockdown or use up economic resources saved. Moreover, the dynamics may differ from what was explained based on the disengagement theory and FBM in the shortterm, leading to a more or less compelling changes in behaviors. We, therefore, concede that focusing on short-term changes in this study is a major shortcoming that future researchers should address. This said, further studies may adopt randomized longitudinal designs to assess the impact of time on the changes considered in this study as well as their effects on mental health. We are also worried that our sample was not powered and may, as a result, not be representative of the general population. While we believe findings of this study may apply to some settings owing to the normal distribution of our data (Garson 2012; Yap and Sim 2011), it is important for future studies to use representative samples to enhance the generalizability of our results. The replication of this study in new contexts may suffice in situations where the use of a powered or representative sample is not possible. Some segments of the population (e.g. older 
people) who did not use the internet were not included in the sample. With English serving as the sole medium of questionnaire administration, residents with poor English skills may have been underrepresented. Despite these limitations, this study is novel for being the first to assess changes in behaviors that may result from self-isolation during the spread of an epidemic. It does not only provide a foundation for future research but also offers insights into what stakeholders could do to ensure that behavior changes do not compound public health issues accompanied by the spread of an epidemic or a related extreme event. At least, this study makes us to contemplate the need for COVID-19 social distancing measures to be rolled out alongside public education programs for discouraging unhealthy changes in behaviors.

\section{Conclusion}

The study confirms short-term changes in behaviors attributable to COVID-19-related social isolation, with key examples being a reduction in individuals' physical activity time and an increase in sedentary behavior time. Sexual activity and eating frequency have changed in the short-term owing to COVID-19-related social isolation. An increase in sedentary behavior time has made the most compelling negative influence on mental health, which suggests that the biggest decline in mental health in our sample was due to increased sedentariness. The only change in behavior that has a positive influence on mental health is substance use. For the most part, changes in behaviors in the short-term attributable to COVID-19 social isolation were associated with lower mental health scores. These changes in behavior are, therefore, potential public health risks that may compound over time.

Acknowledgements We thank members of the 'Share Research-ACE' WhatsApp team including Dr Samuel Awuni Azinga and Mr Wisdom Mensah Avor for their technical advice and guidance. We acknowledge Hon. Kojo Yankah for proofreading this manuscript.

Author Contributions NA conceived the research project and wrote the manuscript. FFO coordinated data collection and survey administration. EM analysed the data. SWM contributed to survey design, validation, and data collection. HKM and FQ contributed to the design of the survey and formatted the manuscript. All authors read and approved the draft manuscript.

Funding The researchers did not receive funding for this study.

\section{Compliance with Ethical Standards}

Conflict of interest The authors declare that they have no conflict of interest.

Ethical Approval This study was approved by the institutional ethics review board (with code \# 0012020-ACE). The board ensured that participation in the study was voluntary and the study was not harmful to participants.

Informed Consent Every participant provided informed consent before completing the survey.

\section{Appendix 1}

See Table 5.
Table 5 Summary statics on items of mental health and its items

\begin{tabular}{lllcrc}
\hline Variable & $\mathrm{N}$ & Minimum & Maximum & Mean & SD \\
\hline I am often carefree and in good spirits & 616 & 1 & 5 & 3.70 & 1.19 \\
I enjoy my life & 621 & 1 & 5 & 4.01 & 0.90 \\
All in all, I am satisfied with my life & 621 & 1 & 5 & 3.91 & 0.92 \\
In general, I am confident & 616 & 1 & 5 & 4.28 & 0.75 \\
I manage well to fulfil my needs & 621 & 1 & 5 & 4.09 & 0.81 \\
I am in good physical and emotional condition & 621 & 1 & 5 & 4.09 & 0.81 \\
I feel that I am well equipped to deal with life and & 616 & 1 & 5 & 3.94 & 0.89 \\
$\quad$ its difficulties & & & & & \\
Much of what I do brings me joy & 611 & 1 & 5 & 3.93 & 0.79 \\
I am a calm, balanced human being & 621 & 2 & 5 & 4.23 & 0.68 \\
Mental health & 621 & 10 & 45 & 30.12 & 7.25 \\
\hline
\end{tabular}




\section{Appendix 2}

See Table 6.

Table 6 Average mental health scores distribution across categorical predictors

\begin{tabular}{|c|c|c|c|c|c|c|}
\hline \multirow[t]{2}{*}{ Variable } & \multirow[t]{2}{*}{ Levels } & \multirow[t]{2}{*}{$\mathrm{N}$} & \multicolumn{3}{|l|}{ Mean } & \multirow[t]{2}{*}{$95 \% \mathrm{Cl}$} \\
\hline & & & Value & SD & $\mathrm{SE}$ & \\
\hline \multirow[t]{7}{*}{ MPATL } & None & 124 & 33.04 & 7.64 & 0.69 & \pm 2.72 \\
\hline & $<30 \min$ & 45 & 34.11 & 7.68 & 1.14 & \pm 4.61 \\
\hline & $30-60 \mathrm{~min}$ & 73 & 29.51 & 6.50 & 0.76 & \pm 3.03 \\
\hline & $1-3 \mathrm{~h}$ & 174 & 28.97 & 5.77 & 0.44 & \pm 1.73 \\
\hline & $4-6 \mathrm{~h}$ & 75 & 28.27 & 6.41 & 0.74 & \pm 2.95 \\
\hline & $>6 \mathrm{~h}$ & 130 & 28.92 & 8.11 & 0.71 & \pm 2.82 \\
\hline & Total & 621 & 30.12 & 7.25 & 0.29 & \pm 1.14 \\
\hline \multirow[t]{7}{*}{ VPATL } & None & 184 & 32.16 & 7.44 & 0.55 & \pm 2.16 \\
\hline & $<30 \min$ & 65 & 29.38 & 5.76 & 0.71 & \pm 2.86 \\
\hline & $30-60 \mathrm{~min}$ & 144 & 29.50 & 7.07 & 0.59 & \pm 2.33 \\
\hline & $1-3 \mathrm{~h}$ & 158 & 29.56 & 6.75 & 0.54 & \pm 2.12 \\
\hline & $4-6 \mathrm{~h}$ & 35 & 28.00 & 8.42 & 1.42 & \pm 5.78 \\
\hline & $>6 \mathrm{~h}$ & 35 & 28.00 & 8.37 & 1.41 & \pm 5.75 \\
\hline & Total & 621 & 30.12 & 7.25 & 0.29 & \pm 1.14 \\
\hline \multirow[t]{7}{*}{ Sedentary time added } & None & 114 & 40.57 & 2.74 & 0.26 & \pm 1.02 \\
\hline & $<30 \min$ & 35 & 38.86 & 3.93 & 0.66 & \pm 2.70 \\
\hline & $30-60 \mathrm{~min}$ & 49 & 33.22 & 3.50 & 0.50 & \pm 2.01 \\
\hline & $1-3 \mathrm{~h}$ & 153 & 29.69 & 4.18 & 0.34 & \pm 1.33 \\
\hline & $4-6 \mathrm{~h}$ & 150 & 25.57 & 3.58 & 0.29 & \pm 1.15 \\
\hline & $>6 \mathrm{~h}$ & 120 & 22.63 & 2.22 & 0.20 & \pm 0.80 \\
\hline & Total & 621 & 30.12 & 7.25 & 0.29 & \pm 1.14 \\
\hline \multirow[t]{3}{*}{ Exercising } & Exercising & 397 & 29.96 & 7.20 & 0.36 & \pm 1.42 \\
\hline & Not exercising & 224 & 30.40 & 7.34 & 0.49 & \pm 1.93 \\
\hline & Total & 621 & 30.12 & 7.25 & 0.29 & \pm 1.14 \\
\hline \multirow[t]{3}{*}{ Smoking frequency } & Unchanged & 173 & 30.39 & 8.07 & 0.61 & \pm 2.42 \\
\hline & Non-smoker & 448 & 30.02 & 6.91 & 0.33 & \pm 1.28 \\
\hline & Total & 621 & 30.12 & 7.25 & 0.29 & \pm 1.14 \\
\hline \multirow[t]{4}{*}{ Alcohol intake } & Increased & 19 & 32.47 & 3.84 & 0.88 & \pm 3.70 \\
\hline & Unchanged & 164 & 31.07 & 8.19 & 0.64 & \pm 2.53 \\
\hline & Not applicable & 438 & 29.67 & 6.94 & 0.33 & \pm 1.30 \\
\hline & Total & 621 & 30.12 & 7.25 & 0.29 & \pm 1.14 \\
\hline \multirow[t]{4}{*}{ Eating frequency } & Decreased & 75 & 29.00 & 6.44 & 0.74 & \pm 2.96 \\
\hline & Increased & 262 & 29.61 & 7.26 & 0.45 & \pm 1.77 \\
\hline & No change & 284 & 30.89 & 7.38 & 0.44 & \pm 1.72 \\
\hline & Total & 621 & 30.12 & 7.25 & 0.29 & \pm 1.14 \\
\hline \multirow[t]{3}{*}{ Substance use } & No & 571 & 30.09 & 7.24 & 0.30 & \pm 1.19 \\
\hline & Yes & 50 & 30.50 & 7.36 & 1.04 & \pm 4.18 \\
\hline & Total & 621 & 30.12 & 7.25 & 0.29 & \pm 1.14 \\
\hline \multirow[t]{4}{*}{ Sexual activity } & Decreased & 125 & 30.16 & 8.27 & 0.74 & \pm 2.93 \\
\hline & Increased & 77 & 33.35 & 7.62 & 0.87 & \pm 3.46 \\
\hline & No change & 419 & 29.52 & 6.69 & 0.33 & \pm 1.29 \\
\hline & Total & 621 & 30.12 & 7.25 & 0.29 & \pm 1.14 \\
\hline \multirow[t]{3}{*}{ Domestic violence increase } & No & 571 & 30.28 & 7.26 & 0.30 & \pm 1.19 \\
\hline & Yes & 50 & 28.30 & 6.94 & 0.98 & \pm 3.95 \\
\hline & Total & 621 & 30.12 & 7.25 & 0.29 & \pm 1.14 \\
\hline \multirow[t]{3}{*}{ CDS } & None & 556 & 30.21 & 7.12 & 0.30 & \pm 1.19 \\
\hline & $1+$ & 65 & 29.38 & 8.28 & 1.03 & 4.10 \\
\hline & Total & 621 & 30.12 & 7.25 & 0.29 & \pm 1.14 \\
\hline \multirow[t]{3}{*}{ Gender } & Female & 215 & 30.28 & 7.83 & 0.53 & \pm 2.10 \\
\hline & Male & 406 & 30.04 & 6.93 & 0.34 & \pm 1.35 \\
\hline & Total & 621 & 30.12 & 7.25 & 0.29 & \pm 1.14 \\
\hline
\end{tabular}

$S D$ standard deviation, $S E$ standard error, $C I$ confidence interval, $C D S$ chronic disease status, $M P A T L$ moderate physical activity time lost, VPATL vigorous physical activity time lost 


\section{References}

Allen, J., Balfour, R., Bell, R., \& Marmot, M. (2014). Social determinants of mental health. International Review of Psychiatry, 26(4), 392-407. https://doi.org/10.1176/appi.focus.20150017.

Anderson, R. M., Heesterbeek, H., Klinkenberg, D., \& Hollingsworth, T. D. (2020). How will country-based mitigation measures influence the course of the COVID-19 epidemic? The Lancet, 395, 391-395. https://doi.org/10.1016/S0140-6736(20)30567-5.

Armitage, R., \& Nellums, L. B. (2020). COVID-19 and the consequences of isolating the elderly. The Lancet Public Health. https ://doi.org/10.1016/s2468-2667(20)30061-x.

Arreola, R., Quintero-Fabián, S., López-Roa, R. I., Flores-Gutiérrez, E. O., Reyes-Grajeda, J. P., Carrera-Quintanar, L., et al. (2015). Immunomodulation and anti-inflammatory effects of garlic compounds. Journal of Immunology Research, 2015, 1-13. https://doi. org/10.1155/2015/401630.

Balhara, Y. P. S., \& Verma, R. (2014). A review of web based interventions focusing on alcohol use. Annals of Medical and Health Sciences Research, 4(4), 472-480. https://doi.org/10.4103/21419248.139272.

Bennett, D. L. (2000). Adolescent mental health and risky sexual behaviour. British Medical Journal, 321(7256), 251-252. https ://doi.org/10.1136/bmj.321.7256.251.

Brooks, S. K., Webster, R. K., Smith, L. E., Woodland, L., Wessely, S., Greenberg, N., et al. (2020). The psychological impact of quarantine and how to reduce it: Rapid review of the evidence. The Lancet, 395, 913-920. https://doi.org/10.1016/S0140-6736(20)30460 $-8$.

Chen, P., Mao, L., Nassis, G. P., Harmer, P., Ainsworth, B. E., \& Li, F. (2020). Opinion Wuhan coronavirus (2019-nCoV): The need to maintain regular physical activity while taking precautions. Journal of Sport and Health Science. https://doi.org/10.1016/j. jshs.2020.02.001.

Chiva-Blanch, G., \& Badimon, L. (2020). Benefits and risks of moderate alcohol consumption on cardiovascular disease: Current findings and controversies. Nutrients, 12, 1-18. https://doi. org/10.3390/nu12010108.

Cumming, E., \& Henry, W. E. (1961). Growing old (p. 227). New York: Basic.

Ellingson, L. D., Meyer, J. D., Shook, R. P., Dixon, P. M., Hand, G. A., Wirth, M. D., et al. (2018). Changes in sedentary time are associated with changes in mental wellbeing over 1 year in young adults. Preventive Medicine Reports, 11, 274-281. https://doi. org/10.1016/j.pmedr.2018.07.013.

Fisher, J., Languilaire, J., Lawthom, R., Nieuwenhuis, R., Petts, R. J., Runswick-Cole, K., et al. (2020). Community, work, and family in times of COVID-19. Community, Work \& Family, 23(3), 247-252. https://doi.org/10.1080/13668803.2020.1756568.

Fogg, B. J. (2009). A behavior model for persuasive design. Proceedings of the 4th International Conference on Persuasive Technology. Persuasive '09 (pp. 401-407). New York, NY, US: ACM.

Fuhrman, J. (2018). The hidden dangers of fast and processed food. American Journal of Lifestyle Medicine, 12(5), 375-381. https:// doi.org/10.1177/1559827618766483.

Galinsky, A. M., \& Waite, L. J. (2014). Sexual activity and psychological health as mediators of the relationship between physical health and marital quality. The Journals of Gerontology Series B: Psychological Sciences and Social Sciences, 69(3), 482-492. https ://doi.org/10.1093/geronb/gbt165.

Ganong, K., \& Larson, E. (2011). Intimacy and belonging. Society and Mental Health, 1(3), 153-172. https://doi.org/10.1177/21568 69311431612
Garson, D. (2012). Testing statistical assumptions (pp. 1-52). Statistical Associates Publishing-Blue Book Publishing, 2012 Edition, NC, U.S.A.

German, J. B., \& Walzem, R. L. (2000). The health benefits of wine. Annual Review of Nutrition, 20(1), 561-593. https://doi. org/10.1146/annurev.nutr.20.1.561.

Hoare, E., Milton, K., Foster, C., \& Allender, S. (2016). The associations between sedentary behavior and mental health among adolescents: A systematic review. International Journal of Behavioral Nutrition and Physical Activity, 13(1), 1-22. https://doi. org/10.1186/s12966-016-0432-4.

Jakobsson, J., Malm, C., Furberg, M., Ekelund, U., \& Svensson, M. (2020). Physical activity during the coronavirus (COVID-19) pandemic: Prevention of a decline in metabolic and immunological functions. Frontiers in Sports and Active Living, 2, 1-4. https:// doi.org/10.3389/fspor.2020.00057.

Johns Hopkins University. (2020). COVID-19 information. Retrieved on 10th April, 2020 at 2:12 PM from https://hub.jhu.edu/novel -coronavirus-information/.

Jones-Jang, S. M., Mortensen, T., \& Liu, J. (2019). Does media literacy help identification of fake news? Information literacy helps, but other literacies don't. American Behavioral Scientist, O(0), 1-18. https://doi.org/10.1177/0002764219869406.

Josefsberg, J. O., \& Buckland, B. (2012). Vaccine process technology. Biotechnology and Bioengineering, 109(6), 1443-1460. https:// doi.org/10.1002/bit.24493.

Kaplan, N. M., Palmer, B. F., \& Denke, M. A. (2000). Nutritional and health benefits of beer. The American Journal of the Medical Sciences, 320(5), 320-326. https://doi.org/10.1097/00000441-20001 1000-00004.

Lawrence, D., Mitrou, F., \& Zubrick, S. R. (2009). Smoking and mental illness: Results from population surveys in Australia and the United States. BMC Public Health, 9(1), 1-11. https://doi. org/10.1186/1471-2458-9-285.

Lewnard, J. A., \& Lo, N. C. (2020). Scientific and ethical basis for social-distancing interventions against COVID-19. The Lancet Infectious Diseases. https://doi.org/10.1016/s1473-3099(20)30190 $-0$.

Li, J., \& Siegrist, J. (2012). Physical activity and risk of cardiovascular disease-A meta-analysis of prospective cohort studies. International Journal of Environmental Research and Public Health, 9(2), 391-407. https://doi.org/10.3390/ijerph9020391.

Liang, H., Xue, Y., \& Berger, B. A. (2006). Web-based intervention support system for health promotion. Decision Support Systems, 42(1), 435-449. https://doi.org/10.1016/j.dss.2005.02.001.

Lin, Q., Zhao, S., Gao, D., Lou, Y., Yang, S., Musa, S. S., et al. (2020). A conceptual model for the outbreak of Coronavirus disease 2019 (COVID-19) in Wuhan, China with individual reaction and governmental action. International Journal of Infectious Diseases. https://doi.org/10.1016/j.ijid.2020.02.058.

Lindberg, M. L., \& Amsterdam, E. A. (2008). Alcohol, wine, and cardiovascular health. Clinical Cardiology, 31(8), 347-351.

Lukat, J., Margraf, J., Lutz, R., van der Veld, W. M., \& Becker, E. S. (2016). Psychometric properties of the Positive Mental Health Scale (PMH-scale). BMC Psychology, 4(1), 1-14. https://doi. org/10.1186/s40359-016-0111-x.

Lund, C., Brooke-Sumner, C., Baingana, F., Baron, E. C., Breuer, E., Chandra, P., et al. (2018). Social determinants of mental disorders and the Sustainable Development Goals: A systematic review of reviews. The Lancet Psychiatry, 5(4), 357-369. https://doi. org/10.1016/S2215-0366(18)30060-9.

Malcolm, M., Frost, H., \& Cowie, J. (2019). Loneliness and social isolation causal association with health-related lifestyle risk in older adults: A systematic review and meta-analysis protocol. Systematic Reviews, 8(1), 1-8. https://doi.org/10.1186/s1364 3-019-0968-x. 
Martin, S. A. (2015). A framework to understand the relationship between social factors that reduce resilience in cities: Application to the City of Boston. International Journal of Disaster Risk Reduction, 12, 53-80. https://doi.org/10.1016/j.ijdrr.2014.12.001.

Mata-Lima, H., Alvino-Borba, A., Pinheiro, A., Mata-Lima, A., \& Almeida, J. A. (2013). Impacts of natural disasters on environmental and socio-economic systems: What makes the difference? Ambiente \& Sociedade, 16(3), 45-64. https://doi.org/10.1590/ S1414-753X2013000300004.

Merolli, M., Sanchez, F. J. M. \& Gray, K. (2014). Social media and online survey: Tools for knowledge management in health research. In Proceedings of the Seventh Australasian Workshop on Health Informatics and Knowledge Management (HIKM 2014), Auckland, New Zealand (pp. 21-29).

Mytton, O. T., Townsend, N., Rutter, H., \& Foster, C. (2012). Green space and physical activity: An observational study using Health Survey for England data. Health \& Place, 18(5), 1034-1041. https ://doi.org/10.1016/j.healthplace.2012.06.003.

Percival, S. S. (2016). Aged garlic extract modifies human immunity. The Journal of Nutrition, 146(2), 433-436. https://doi. org/10.3945/jn.115.210427.

Prentice, A. M. (2001). Overeating: The health risks. Obesity Research, 9(11), 234-238. https://doi.org/10.1038/oby.2001.124.

Pung, R., Chiew, C. J., Young, B. E., Chin, S., Chen, M. I.-C., Clapham, H. E., et al. (2020). Investigation of three clusters of COVID-19 in Singapore: Implications for surveillance and response measures. The Lancet, 395, 1039-1046. https://doi.org/10.1016/S0140 -6736(20)30528-6.

Regmi, P. R., Waithaka, E., Paudyal, A., Simkhada, P., \& Van Teijlingen, E. (2017). Guide to the design and application of online questionnaire surveys. Nepal Journal of Epidemiology, 6(4), 640-644. https://doi.org/10.3126/nje.v6i4.17258.
Rezai, M., Côté, P., Cassidy, J. D., \& Carroll, L. (2008). The association between prevalent neck pain and health-related quality of life: A cross-sectional analysis. European Spine Journal, 18(3), 371-381. https://doi.org/10.1007/s00586-008-0823-6.

Rothman, K. J., \& Greenland, S. (1998). Modern epidemiology (2nd ed.). Philadelphia: Lippincott-Raven.

Sederer, L. I. (2016). The social determinants of mental health. Psychiatric Services, 67(2), 234-235.

Serafini, G., Parmigiani, B., Amerio, A., Aguglia, A., Sher, L., \& Amore, M. (2020). The psychological impact of COVID-19 on the mental health in the general population. QJM: An International Journal of Medicine, 113(8), 531-537. https://doi.org/10.1093/ qjmed/hcaa201.

Shim, R., Koplan, C., Langheim, F. J. P., Manseau, M. W., Powers, R. A., \& Compton, M. T. (2014). The social determinants of mental health: An overview and call to action. Psychiatric Annals, 44(1), 22-26. https://doi.org/10.3928/00485713-20140108-04.

Vindegaard, N., \& Eriksen Benros, M. (2020). COVID-19 pandemic and mental health consequences: Systematic review of the current evidence. Brain, Behavior, and Immunity, 67, 1-12. https://doi. org/10.1016/j.bbi.2020.05.048.

Walsh, R. (2011). Lifestyle and mental health. American Psychologist, 66(7), 579-592. https://doi.org/10.1037/a0021769.

Yap, B. W., \& Sim, C. H. (2011). Comparisons of various types of normality tests. Journal of Statistical Computation and Simulation, 81(12), 2141-2155. https://doi.org/10.1080/00949 655.2010 .520163 .

Publisher's Note Springer Nature remains neutral with regard to jurisdictional claims in published maps and institutional affiliations. 